\title{
Contribución energética de la digestión anaerobia al proceso de producción de biodiesel a partir de microalgas
}

\author{
Energetic contribution of anaerobic digestion to the production process \\ of biodiesel from microalgae
}

Patricio Neumann $^{1} \quad$ David Jeison $^{1}$

Recibido 7 de enero de 2013, aceptado 17 de julio de 2014

Received: January 7, 2014 Accepted: July 17, 2014

\begin{abstract}
RESUMEN
La producción de biodiesel, una de las alternativas propuestas para enfrentar la crisis energética actual, se ha visto fuertemente cuestionada debido a que las materias primas actualmente utilizadas resultan competitivas con los suelos destinados para la producción de alimentos. El uso de microalgas como materia prima permitiría superar tal barrera, al tiempo que su cultivo implica ventajas tales como mayores productividades de lípidos trans-esterificables y mayores tasas de fijación de $\mathrm{CO}_{2}$. Sin embargo, según estudios realizados su implementación implicaría rendimientos energéticos deficientes e incluso negativos. El presente trabajo consta de una evaluación de los principales beneficios de la digestión anaerobia como estrategia de recuperación de energía a partir de la biomasa residual del proceso de producción de biodiesel. La generación de energía en forma de biogás fue comparada con la energía generada por el biodiesel, evaluando distintos escenarios relativos a 2 variables de la biomasa, la acumulación de lípidos y la biodegradabilidad anaerobia del residuo. Los resultados indican un importante potencial en el biogás, prácticamente equivalente a la energía producida en forma de biodiesel. Se destaca el elevado potencial energético del proceso, pero se recalca a su vez que este es dependiente de factores tales como el porcentaje de acumulación de lípidos trans-esterificables en las microalgas, la productividad de la biomasa y la biodegradabilidad alcanzada durante la digestión del residuo.
\end{abstract}

Palabras clave: Biodiesel, microalgas, digestión anaerobia, biogás, bioenergías.

\section{ABSTRACT}

Biodiesel production, one of the alternatives proposed to overcome the energetic crisis, has been strongly criticized because the feedstocks currently used compete with food production for fertile soils. The use of microalgae as feedstock would allow to overcome that barrier and its use involves advantages such as higher trans-esterifiable lipids accumulation and higher $\mathrm{CO}_{2}$ fixation rates. However, according to recent studies, its implementation would imply deficient and even negative energetic yields. The present work consists of an evaluation of the principal energetic benefits of anaerobic digestion as an energy recovery strategy from the biomass residues of the biodiesel production processes. The energy generation in the form of biogas was compared with the energy generated by the biodiesel, evaluating different scenarios related to 2 variables of the biomass, lipid accumulation and anaerobic biodegradability of the residue. The results show an important potential of the generated biogas, practically equal to the energy produced as biodiesel. The high energetic potential of the process is highlighted, but also the fact that it depends on factors like the accumulation of lipids suitable for trans-esterification in the microalgae, the biomass productivity and the degree of biodegradation achieved during the digestion of the residue.

Keywords: Biodiesel, microalgae, anaerobic digestion, biogas, bioenergy.

1 Departamento de Ingeniería Química. Universidad de La Frontera. Av. Francisco Salazar 01145. Temuco, Chile. E-mail: p.neumann01@ufromail.cl; djeison@ufro.cl 


\section{INTRODUCCIÓN}

\section{Antecedentes generales}

Durante los últimos años, el mundo ha debido enfrentarse a una incipiente crisis energética, gatillada por un creciente aumento en el consumo energético y por la incapacidad de suplir tal demanda por medio de las fuentes fósiles tradicionales. Durante el último cuarto de siglo, la demanda mundial de energía se incrementó en aproximadamente un $60 \%$, y la mayor parte de los pronósticos para los próximos veinticinco años proyectan un porcentaje de aumento similar [14]. Considerando que el $80 \%$ de la matriz energética mundial está conformada por petróleo, gas natural y carbón, y que la producción de estos se encuentra actualmente en su tope máximo [14], la búsqueda de fuentes de energías renovables alternativas se vuelve imprescindible para el desarrollo sustentable de la sociedad.

Afortunadamente, nuestro planeta dispone de un sinnúmero de recursos energéticos potencialmente aprovechables por el hombre, entre los que destacan las energías eólica, solar, hidráulica, mareomotriz y las bioenergías. Estas últimas corresponden a aquellas tecnologías que aprovechan de manera directa o indirecta la biomasa vegetal, por lo que en esencia consisten en energía solar capturada mediante la fotosíntesis y almacenada como energía química en los tejidos de los vegetales. La biomasa representa en la actualidad el 10\% de la producción primaria de energía a nivel mundial, lo que la ubica en cuarto lugar tras el petróleo, carbón y gas natural, y la convierte en la fuente de energía renovable más importante [15].

De los dos biocombustibles líquidos principales (biodiesel y bioetanol), el biodiesel es probablemente el que ha recibido mayor atención los últimos años debido a que representa una alternativa directa y de fácil transición desde el diesel tradicional para los motores de combustión interna.

El biodiesel corresponde a la mezcla de compuestos orgánicos obtenida mediante la trans-esterificación de triglicéridos contenidos en aceites vegetales. Dentro de las fuentes de los aceites utilizados como materia prima para la producción de biodiesel destacan cultivos como el raps (Brassica napus), cuya semilla acumula lípidos en cantidades significativas. Sin embargo, este tipo de cultivos utilizan suelos productivos dedicados a la siembra de cereales y otros cultivos con fines alimentarios. Debido a esto, la producción de biodiesel a partir de estas fuentes ha sido fuertemente cuestionada, existiendo controversia acerca de la disponibilidad de suelos para la obtención de biodiesel sin afectar la producción actual de alimentos.

En este escenario, el encontrar fuentes alternativas de lípidos trans-esterificables se ha vuelto un eje trascendental para el desarrollo de una tecnología de biodiesel sustentable. Dentro de las alternativas propuestas durante los últimos años destaca el potencial uso de microalgas. Las microalgas presentan ventajas tales como sus altos rendimientos de producción de aceites, que superan ampliamente a los obtenidos con cultivos tradicionales [5], sus mayores tasas de fijación de $\mathrm{CO}_{2}$ que podrían utilizarse para la mitigación de la concentración atmosférica de este gas [22], y su independencia de suelos fértiles. Además, el biodiesel obtenido de esta fuente posee mejores características técnicas que el biodiesel de otras fuentes vegetales, en términos de una menor viscosidad y densidad y una mayor estabilidad química [4].

En términos de rendimientos, se estima que con productividades de biodiesel de alrededor de $121.104 \mathrm{~kg} \mathrm{ha}^{-1} \mathrm{año}^{-1}$ [6], el aceite proveniente de 8-12 millones de hectáreas de cultivos de microalgas reemplazaría toda la importación de petróleo de EE.UU., dejando alrededor de 180 millones de hectáreas fértiles libres para la producción de alimentos [21].

\section{Limitaciones técnicas y económicas de la producción de biodiesel a partir de microalgas}

A pesar de lo promisorio resulta la producción de biodiesel a partir de microalgas como alternativa de producción de energía limpia y renovable, el proceso presenta ciertas limitaciones técnicas. Estas están asociadas principalmente a su rendimiento energético, ya que debido al extensivo uso de energía de ciertas etapas tales como la cosecha, secado de la biomasa y extracción de lípidos [16], en las condiciones actuales el proceso presenta un bajo e incluso negativo balance energético [10].

Una de las aproximaciones propuestas para abordar el problema del bajo rendimiento energético del 
proceso consiste en acoplar un reactor de digestión anaerobia de la biomasa residual post-extracción de lípidos al proceso de producción de biodiesel [16], de manera de recuperar la energía contenida en tal residuo en forma de biogás. La digestión anaerobia es una tecnología robusta, ampliamente utilizada para la estabilización de residuos y la producción de energía desde fines del siglo XIX [12], por lo que en principio existiría factibilidad técnica para su aplicación en el esquema propuesto.

\section{Digestión anaerobia como alternativa para} mejorar el rendimiento energético del proceso

La digestión anaerobia es un proceso durante el cual la materia orgánica es convertida por acción bacteriana en una mezcla gaseosa. Esto ocurre en ausencia de oxígeno u otros aceptores inorgánicos de electrones, condiciones en las cuales la materia orgánica es utilizada tanto como dador como aceptor de electrones. El producto de este proceso es una mezcla gaseosa compuesta principalmente por metano y dióxido de carbono.

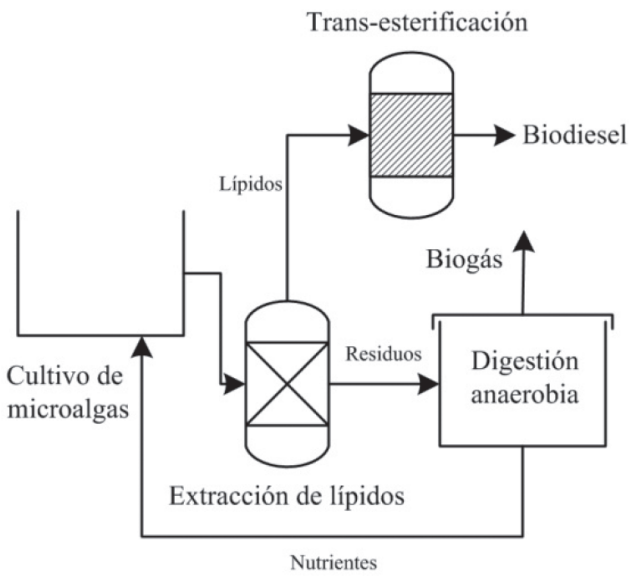

Figura 1. Diagrama del proceso estudiado.

La Figura 1 muestra el esquema propuesto, en el que se incluye la digestión anaerobia de la biomasa residual.

Estudios realizados por Lardon y colaboradores [10] determinaron que por cada MJ de energía producido en el biodiesel, existen 1,23 MJ de energía en el residuo generado. Esta energía puede recuperarse mediante digestión anaerobia, generando biogás. Además, la degradación de los compuestos presentes en el residuo resulta en la producción de un fertilizante nitrogenado, que podría utilizarse como fuente de nutrientes durante el cultivo de las microalgas [16].

\section{MATERIALES Y MÉTODOS}

\section{Potencial energético de la digestión anaerobia de la biomasa residual}

Se realizó una estimación del potencial energético del biodiesel y del biogás generado en un hipotético proceso de producción integrado de ambos biocombustibles. Se utilizó una caracterización genérica de la composición de la microalga, suponiendo una acumulación de lípidos elevada, apta para la producción de biodiesel (30\% en peso seco), y porcentajes de proteínas y carbohidratos complementarias a esta ( $45 \%$ y $25 \%$ en peso seco, respectivamente). Se utilizó un poder calorífico del biodiesel igual a $40 \mathrm{MJ} \mathrm{kg}^{-1}$ y la generación de metano a partir de cada componente (proteínas, carbohidratos y lípidos) se estimó según los factores específicos reportados por Sialve y colaboradores [17].

Los resultados obtenidos se extrapolaron a un esquema productivo que consideraba la utilización del biogás para generación de energía eléctrica y térmica (co-generación), suponiendo un rendimiento de un 33\% para la generación de energía eléctrica y una recuperación de un $50 \%$ de la energía térmica restante. Se supuso la utilización de esta última para la recuperación del solvente que se usaría en la etapa de extracción de lípidos (hexano a una temperatura de entrada de $25^{\circ} \mathrm{C}$ ).

Análisis de sensibilidad de la generación energética Se realizó un análisis de sensibilidad del potencial energético del proceso frente a variaciones en la biodegradabilidad anaerobia del residuo microalgal y en la acumulación de lípidos en la biomasa, debido a que estas dos variables tienen un efecto potencial significativo en el balance energético global. El análisis de regresión fue realizado con el software Minitab 15.

\section{Cuantificación de los flujos de energía y masa del proceso}

Se realizaron balances de masa y energía de manera de poder determinar las entradas y salidas del proceso. Se utilizó como base de cálculo una hectárea de cultivo de microalgas, basándose en un valor de productividad adaptado de Chisti [5] Se supuso un $30 \%$ de acumulación de lípidos en la 
biomasa [11] y una biodegradabilidad del residuo de un $100 \%$, comparándose la energía generada a partir del residuo microalgal con la energía que se obtiene por concepto del biodiesel.

\section{RESULTADOS Y DISCUSIÓN}

\section{Potencial energético de la digestión de la biomasa residual}

Se determinó el potencial energético de un proceso de producción de biocombustibles a partir de microalgas, específicamente la producción conjunta de biodiesel y biogás. La Figura 2 presenta el potencial energético bruto en base a las estimaciones realizadas, mientras la Figura 3 muestra la distribución porcentual que tendrían los flujos energéticos si la energía recuperada en el biogás se utilizara en un esquema de co-generación y recuperación de solvente.

A partir de estos resultados, es posible apreciar el importante potencial energético que tendría la digestión anaerobia de la biomasa residual dentro del esquema propuesto. La suma de la energía eléctrica y la energía térmica que potencialmente se pueden generar por este medio equivale a prácticamente el $100 \%$ de la energía contenida en el biodiesel, aún considerando que los lípidos extraídos previamente representan la fracción con

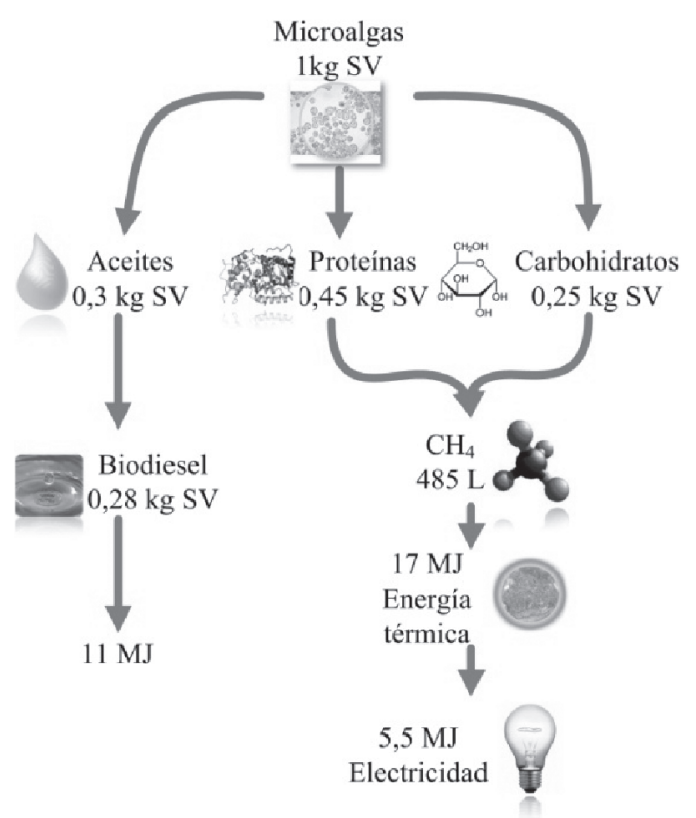

Figura 2. Generación bruta de energía a partir de 1 $\mathrm{kg}$ de sólidos volátiles (SV) de microalgas. mayor potencial energético de la biomasa, debido a su estado químico altamente reducido.

Tal como se aprecia en la Figura 3, la energía térmica recuperable a partir de la co-generación resulta suficiente para suplir los requerimientos de la etapa de recuperación de solvente, una de las etapas más demandantes en energía del proceso, dejando un excedente de energía térmica que representa alrededor de un $9 \%$ de la energía total. De esta manera la digestión anaerobia del residuo puede jugar un papel esencial en el esquema productivo de biodiesel a partir de microalgas, ya sea que la energía térmica generada se utilice para los fines aquí propuestos o en otras etapas del proceso tales como el secado de la biomasa previo a la transesterificación (de ser necesario). Aún más, desde el punto de vista energético metanizar íntegramente la microalga resulta una alternativa competitiva con el proceso combinado biodiesel/biogás, ya que la conversión de los lípidos presentes en la biomasa a biogás permitiría obtener alrededor de 10,65 MJ de energía por kg SV de microalgas. De esta forma, la selección de un proceso sobre otro dependerá en gran parte de las necesidades locales de energía y del mercado objetivo del proceso.

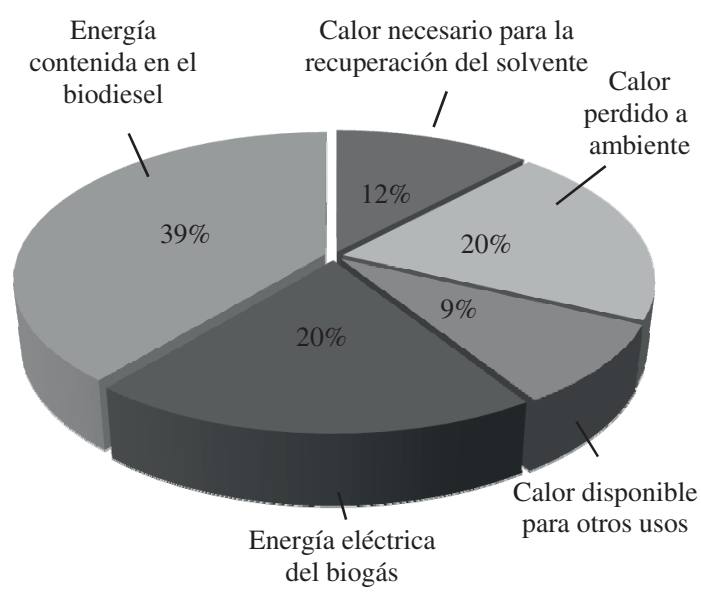

Figura 3. Distribución porcentual de los flujos energéticos estudiados.

\section{Análisis de sensibilidad de la generación de energía}

La Figura 4 muestra los resultados del análisis de sensibilidad realizado. Se estudiaron las variaciones en el potencial energético del proceso frente a variaciones en el porcentaje de acumulación de lípidos de la biomasa y la bio-degradabilidad anaeróbica del residuo microalgal. 


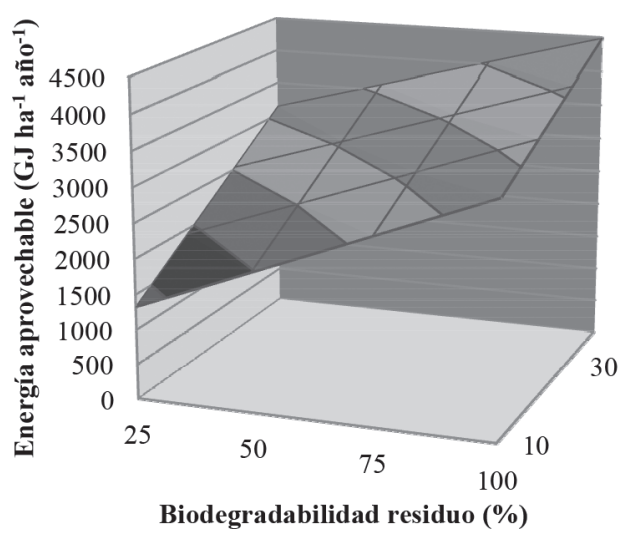

Figura 4. Análisis de sensibilidad de la generación de energía en función de la acumulación de lípidos y biodegradabilidad del residuo.

Estos resultados permiten apreciar que debe considerarse que el potencial energético del biogás y del proceso global son altamente dependientes de variables tales como el nivel de acumulación de lípidos tran-esterificables en las microalgas y el nivel de bio-degradabilidad alcanzado durante la digestión del residuo. Mientras el primer factor afecta directamente a la productividad del biodiesel y por ende al principal producto, el segundo factor afecta la recuperación de energía desde el residuo.

Además, en la Figura 4 se aprecia que la energía aprovechable en el proceso presenta una mayor pendiente frente a cambios en la acumulación de lípidos trans-esterificables que frente a cambios en la biodegradabilidad del residuo, debido al mayor poder calorífico del biodiesel comparado con el biogás. En este escenario, para obtener mejores resultados se recalca la necesidad de optimizar dos factores clave del proceso:

- La selección y el cultivo del alga, con miras a la productividad de lípidos y biodiesel.

- La biodegradabilidad anaeróbica del residuo.

Con respecto al primer punto, existen especies tales como Botryococcus braunii que pueden alcanzar niveles de acumulación de lípidos de hasta un $75 \%$ en peso seco [5] en condiciones de cultivo que incluyen el uso de medios con limitación de nitrógeno [9]. Sin embargo, la tasa de crecimiento y productividad de biomasa tienden a disminuir en tales condiciones [7], siendo importante mantener un compromiso entre productividad de biomasa y acumulación de lípidos para lograr una producción de biodiesel adecuada. Además, es necesario recalcar que los lípidos acumulados por la microalga deben ser trans-esterificables, vale decir deben ser compuestos que puedan ser convertidos en metil ésteres de ácidos grasos (principales constituyentes del biodiesel) en presencia de un alcohol y un catalizador. Ácidos grasos de cadena corta tales como los ácidos palmítico, oleico y linoleico presentes en Botryococcus braunii constituyen ácidos grasos transesterificables [18].

Por otra parte, la bio-degradabilidad del residuo (y por ende el rendimiento de metano) es también dependiente de la especie de microalga utilizada. $\mathrm{La}$ existencia de pared celular y polímeros extracelulares puede ser una limitación para la transferencia de masa del contenido intracelular del residuo hacia las bacterias encargadas de la degradación. Este y otros factores limitantes asociados a la hidrólisis de la materia orgánica de alto peso molecular pueden hacer necesaria la utilización de un proceso de pretratamiento del residuo, que permita la ruptura de los polímeros y la transferencia del material intracelular hacia el medio para su posterior conversión a biogás. Sin embargo, debe existir un compromiso entre la mejora en la biodegradabilidad obtenida y el gasto energético y económico del pre-tratamiento, de manera de asegurar que este sirva realmente a su propósito de mejora energética.

Mediante un análisis de regresión de los resultados (previo a la multiplicación de estos por la productividad de biomasa) se obtuvo una ecuación que permite describir la relación estadística entre las variables independientes acumulación de lípidos y biodegradabilidad del residuo y la variable dependiente generación bruta de energía total, en función de la productividad total de biomasa (ecuación (1)).

$$
E=\frac{(2,28+0,12 B+0,27 L) * P}{1000}
$$

Donde:

$\mathrm{E}=$ Energía $\left(\mathrm{GJ}\right.$ año $\left.{ }^{-1}\right)$

$\mathrm{B}=$ Biodegradabilidad $(\%)$

$\mathrm{L}=$ Acumulación de lípidos (\% peso seco)

$\mathrm{P}=$ Productividad de biomasa $\left(\mathrm{kg}\right.$ año $\left.{ }^{-1}\right)$ 
La ecuación (1) permite realizar estimaciones de la energía producida por unidad productiva (hectárea, metro cúbico, etc.) para cada especie de microalga en función de 3 de las variables más importantes del proceso. Por ejemplo, en un escenario conservador para la especie Botryococcus braunii, se han reportado porcentajes de acumulación de lípidos de alrededor de un 17\% [1], con biodegradabilidades anaerobias del residuo del orden del 70\% [19] y productividades de $0,02 \mathrm{~g} \mathrm{~L}^{-1}$ día $^{-1}$ [3]. En este escenario, la producción de energía total es de alrededor de $3,05 \times 10^{-4} \mathrm{GJ} \mathrm{m}^{-3}$ año $\mathrm{O}^{-1}$ o $3,05 \mathrm{GJ}$ ha $^{-1}$ año $^{-1}$ (suponiendo fotobiorreactores de $1 \mathrm{~m}^{2}$ de superficie), valor que representa alrededor de un $0,07 \%$ de la energía máxima estimada en este trabajo (para $\mathrm{L}=40 \%$ y $\mathrm{B}=100 \%$ ). Es importante señalar la existencia de reportes de otros autores que indican productividades para esta misma especie de microalga de entre $0,114 \mathrm{~g} \mathrm{~L}^{-1}$ día $^{-1}$ [1] a $0,304 \mathrm{~g} \mathrm{~L}^{-1}$ día $^{-1}$ [20] con porcentajes de acumulación de lípidos de hasta un 65\% [20], dependiendo de la configuración de fotobiorreactor utilizada, las condiciones de cultivo y la cepa de Botryococcus braunii cultivada. Esto si bien implica una enorme variabilidad en la energía bruta que se puede obtener del proceso (llegando hasta 85,8 GJ $\mathrm{ha}^{-1} \mathrm{año}^{-1}$ ), en las condiciones actuales esta especie no representa una alternativa para la producción de bioenergía en el esquema biodiesel-biogás, principalmente debido a su baja productividad. Sin embargo, la posibilidad de optimizar tal productividad y la existencia de otras especies de microalgas con productividades de hasta alrededor de 130 ton ha ${ }^{-1}$ año $^{-1}\left(35 \mathrm{~g} \mathrm{~L}^{-1}\right.$ día $\left.{ }^{-1}\right)$ y con niveles de acumulación de lípidos de hasta un 52\% para Nannochloropsis sp. [13], permiten prever un futuro auspicioso para esta tecnología, siempre recalcando la alta dependencia que tiene de las variables estudiadas en este trabajo.

\section{Cuantificación de los flujos de energía y masa del proceso}

La Figura 5 muestra el esquema de producción propuesto, incluyendo el balance de masa y energía de los principales flujos expresados en base a la productividad anual de una hectárea de cultivo de microalgas.

Es posible apreciar que con las productividades de biomasa y lípidos esperados, los flujos energéticos generados por el proceso son considerables. En este escenario, la energía generada por el biodiesel es de 2 millones de $\mathrm{MJ} \mathrm{ha}^{-1}$ año $^{-1}$, equivalente a aproximadamente 327 barriles equivalentes de petróleo (BEP) por hectárea al año, mientras que la potencia eléctrica generada por el biogás sería de alrededor de $33,3 \mathrm{~kW}$, vale decir la energía consumida por alrededor de 97 personas (suponiendo

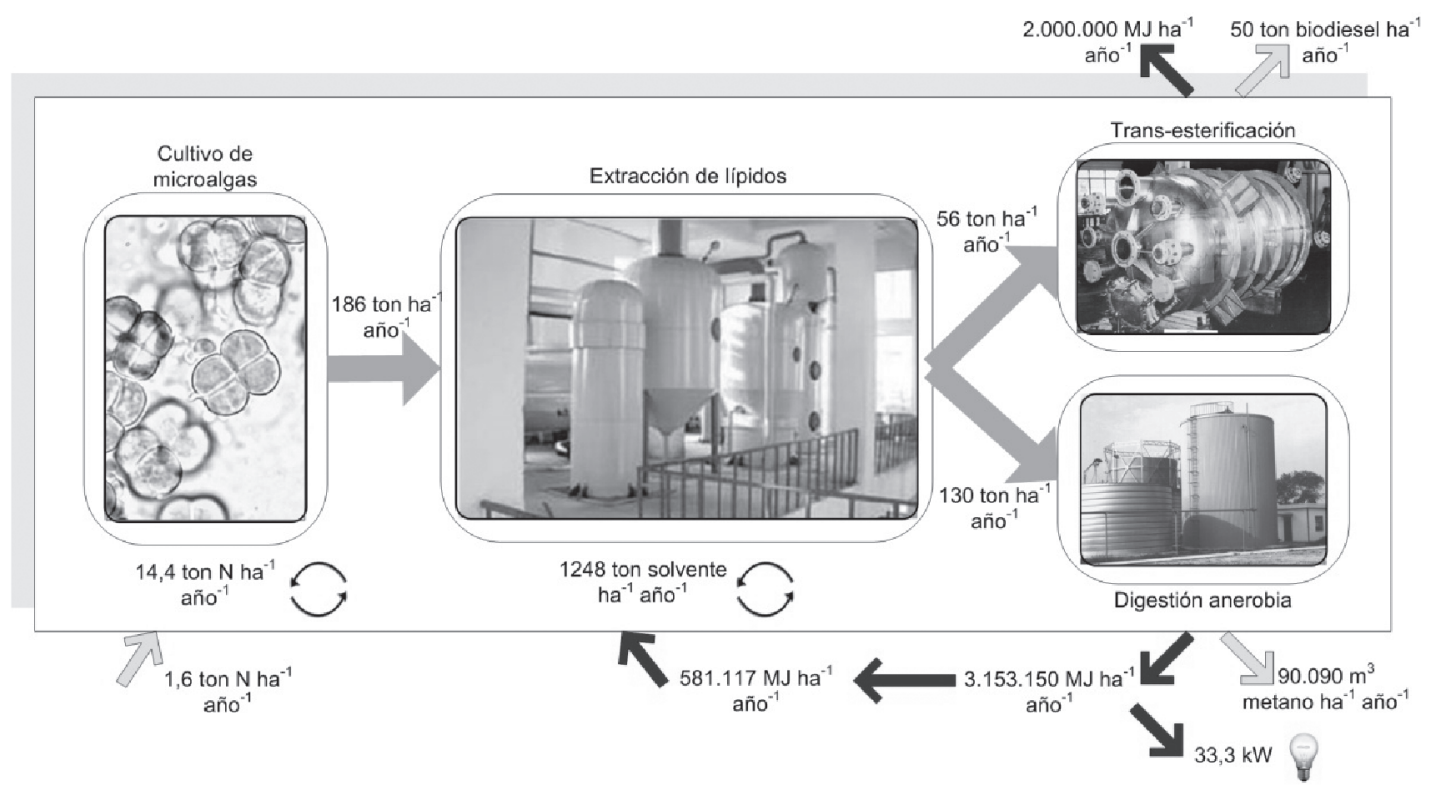

Figura 5. Balance de materia y energía del proceso global. 
un consumo energético per cápita de alrededor de $3.000 \mathrm{~kW}-\mathrm{h}$ anual).

La energía térmica aprovechable generada permitiría recuperar la totalidad del hexano utilizado para la extracción, y si se alcanza una recuperación de nutrientes de un $90 \%$ desde el estanque de digestión, es posible reducir el consumo de nitrógeno en el cultivo en más de 14 toneladas por hectárea al año, existiendo alternativas tecnológicas ad-hoc para la recuperación de este tipo de compuestos disueltos tales como las membranas, estrategia que ha mostrado porcentajes de recuperación de alrededor de un 99\% del amonio presente en matrices complejas tales como aguas residuales $[2,8]$.

\section{CONCLUSIONES}

A partir de estos resultados y de su análisis posterior, surgen los siguientes comentarios y conclusiones:

- Si bien el potencial energético del residuo resulta elevado, este es dependiente de variables que deben ser estudiadas y optimizadas. Tanto la especie de alga así como su cultivo y procesamiento resultan importantes a este respecto.

- Resulta necesario orientar la estrategia de cultivo de las microalgas a la obtención de una elevada productividad de lípidos. Para esto deben estudiarse tanto los factores asociados a la capacidad de acumulación de lípidos de la especie como la productividad de biomasa obtenida, aspectos críticos para el desarrollo y escalamiento de la tecnología.

- Debido a la gran influencia que tienen las variables estudiadas (acumulación de lípidos, biodegradabilidad del residuo y productividad de biomasa) sobre la energía generada por el proceso, la selección de una especie de microalga apta que cumpla con todas las condiciones necesarias representa un factor crítico. Resulta además necesario aunar tal selección con criterios económicos y técnicos relacionados con el procesamiento de la biomasa obtenida.

- El proceso de producción de biocombustibles a partir de microalgas posee un importante potencial para el desarrollo de las ERNC en nuestro país, pero el impacto que este tenga en la matriz energética nacional dependerá en gran medida de lograr alcanzar condiciones adecuadas tanto para el cultivo como para el procesamiento de la biomasa.

\section{REFERENCIAS}

[1] V. Ashokkumar and R. Rengasamy. "Mass culture of Botryococcus braunii Kutz. under open raceway pond for biofuel production". Bioresource Technology. Vol. 104, pp. 394399. November, 2012. ISSN: 0960-8524. URL: http://www.sciencedirect.com/ science/ article/pii/S0960852411015811. DOI: 10.1016/j.biortech.2011.10.093.

[2] S.N. Ashrafizadeh and Z. Khorasani. "Ammonia removal from aqueous solutions using hollow-fiber membrane contactors". Chemical Engineering Journal. Vol. 162, pp. 242-249. August, 2010. ISSN: 13858947. URL: http://www.sciencedirect.com/ science/article/pii/S1385894710004754. DOI: 10.1016/j.cej.2010.05.036.

[3] J. Bazaes, C. Sepúlveda, F. Acién, J. Morales, L. Gonzales, M. Rivas and C. Riquelme. "Outdoor pilot-scale production of Botryococcus braunii in panel reactors". Journal of Applied Phycology. Vol. 24, pp. 1353-1360. February, 2012. ISSN: 0921-8971. URL: http://link.springer.com/ article/ 10.1007\%2Fs10811-012-9787-3. DOI: 10.1007/s10811-012-9787-3.10. 1007/ s10811-012-9787-3.

[4] J.A.V. Costa and M.G. de Morais. "The role of biochemical engineering in the production of biofuels from microalgae". Bioresource Technology. Vol. 102, pp. 2-9. January, 2011. ISSN: 0960-8524. URL: http://www.sciencedirect.com/science/article/ pii/S0960852410009946. DOI: 10.1016/j. biortech.2010.06.014.

[5] Y. Chisti. "Biodiesel from microalgae". Biotechnology Advances. Vol. 25, pp. 294 306. February, 2007. ISSN: 0734-9750. URL: http://www.sciencedirect.com/science/article/ pii/S0734975007000262. DOI: 10.1016/j. biotechadv.2007.02.001.

[6] X. Deng, Y. Li and X. Fei. "Microalgae: A promising feedstock for biodiesel". African Journal of Microbiology Research. Vol. 3, pp. 1008-1014. December, 2009. ISSN: 19960808. URL: http://www.academic journals. 
org/article/article1380378013_Deng\%20 et\%20al.pdf

[7] M.R. Droop. "25 years of algal growth kinetics: A personal view". Botanica Marina. Vol. 26, pp. 99-112. 1983. ISSN: 1437-4323. URL: http://www.degruyter. com/view/j/ botm.1983.26.issue-3/botm. 1983.26.3.99/ botm.1983.26.3.99.xml. DOI: 10.1515/ botm.1983.26.3.99.

[8] A. Hasanoğlu, J. Romero, B. Pérez and A. Plaza. "Ammonia removal from wastewater streams through membrane contactors: Experimental and theoretical analysis of operation parameters and configuration". Chemical Engineering Journal. Vol. 160, pp. 530-537. June, 2010. ISSN: 1385-8947. URL: http://www.sciencedirect.com/ science/ article/pii/S1385894710003001. DOI: 10.1016/j.cej.2010.03.064.

[9] A.M. Illman, A.H. Scragg y S.W. Shales. "Increase in Chlorella strains calorific values when grown in low nitrogen medium". Enzyme and Microbial Technology. Vol. 27, pp. 631-635. November, 2000. ISSN: 01410229.URL: http://www.sciencedirect.com/ science/article/pii/S0141022900002660. DOI: 10.1016/S0141-0229(00)00266-0

[10] L. Lardon, A. Hélias, B. Sialve, J.-P. Steyer and O. Bernard. "Life-Cycle Assessment of Biodiesel Production from Microalgae". Environmental Science \& Technology. Vol. 43, pp. 6475-6481. July, 2009. ISSN: 0013-936X. URL. http://pubs.acs.org/doi/ abs/ 10.1021/es900705j. DOI: 10.1021/ es900705j.10.1021/es900705j.

[11] T.M. Mata, A.C. Melo, S. Meireles and A. M. Mendes. "Potential of Microalgae Scenedesmus obliquus Grown in Brewery Wastewater for Biodiesel Production". Chemical Engineering Transactions. Vol. 32, pp. 901-906. June, 2013. ISSN: 1974-9791. URL: http://www.aidic.it/cet/13/32/151.pdf. DOI: 10.3303/ CET1332151

[12] P.L. McCarty. "The development of anaerobic treatment and its future". Water Science \& Technology. Vol. 44, pp. 149-156. 2001. ISSN: 0273-1223 URL: http://www.iwaponline. com/wst/04408/wst044080149.htm

[13] N. Moazami, A. Ashori, R. Ranjbar, M. Tangestani, R. Eghtesadi and A. S. Nejad. "Large-scale biodiesel production using microalgae biomass of Nannochloropsis". Biomass and bioenergy. Vol. 39, pp. 449453. April, 2012. ISSN: 0961-9534. URL: http://www.sciencedirect.com/science/article/ pii/S0961953412000669. DOI: 10.1016/ j.biombioe.2012.01.046.

[14] L. Raymond, A. Gould, J. Hamre, D. O'Reilly y D. Yergin. "Enfrentando el grave problema energético". National Petroleum Council. Washington D.C., USA, pp. 7-8. Fecha de actualización: 18 de julio de 2012. Fecha de consulta: 15 de noviembre de 2012. URL: http://www.npc.org/hard_truths-translations/ hardtruths_s spanish.pdf

[15] J. Sawin. Renewables 2013: Global Status Report, p. 178. Renewable Energy Policy Network for the 21st Century. Paris, pp. 27-33. Date of visit: November 15, 2012. URL: http://www.ren21.net/portals/0/ documents/ resources/gsr/2013/gsr2013_lowres.pdf

[16] S.A. Scott, M.P. Davey, J.S. Dennis, I. Horst, C.J. Howe, D.J. Lea-Smith and A.G. Smith. "Biodiesel from algae: challenges and prospects". Current Opinion in Biotechnology. Vol. 21, pp. 277-286. June, 2010. ISSN: 0958-1669. URL: http://www.sciencedirect. com/science/article/pii/S0958166910000443. DOI: 10.1016/j.copbio.2010.03.005.

[17] B. Sialve, N. Bernet and O. Bernard. "Anaerobic digestion of microalgae as a necessary step to make microalgal biodiesel sustainable". Biotechnology Advances. Vol. 27, pp. 409-416. July-August, 2009. ISSN: 0734-9750. URL: http://www. sciencedirect. com/science/article/pii/ S0734975009000457. DOI: $10.1016 / \mathrm{j}$. biotechadv.2009.03.001.

[18] E.B. Sydney, T.E. da Silva, A. Tokarski, A.C. Novak, J.C. de Carvalho, A.L. Woiciecohwski, C. Larroche and C.R. Soccol. "Screening of microalgae with potential for biodiesel production and nutrient removal from treated domestic sewage". Applied Energy. Vol. 88, pp. 3291-3294. October, 2011. ISSN: 03062619. URL: http://www.sciencedirect.com/ science/article/pii/S0306261910004873. DOI: 10.1016/j.apenergy.2010.11.024.

[19] A. Torres, P. Neumann, L. Meier, L. Azócar, M. Rivas and D. Jeison. "Biogas production potential and ammonium release in anaerobic digestion of spent microalgae". III Congreso 
Latinoamericano Biorrefinerías. Pucón, Chile. 19-21 noviembre de 2012.

[20] H.-L. Tran, J.-S. Kwon and C.-G. Lee. "Optimization for the growth and the lipid productivity of Botryococcus braunii LB572". Journal of Bioscience and Bioengineering. Vol. 108, pp. S55. November, 2009. ISSN: 1389-1723. URL: http://www.sciencedirect. com/science/article/pii/S1389172309005386. DOI: 10.1016/ j.jbiosc.2009.08.157.

[21] B.-H. Um and Y.-S. Kim. "Review: A chance for Korea to advance algal-biodiesel technology". Journal of Industrial and
Engineering Chemistry. Vol. 15, pp. 1-7. January, 2009. ISSN: 1226-086X. URL: http://www.sciencedirect.com/science/article/ pii/S1226086X09000240. DOI: http://dx.doi. org/10.1016/j.jiec.2008.08.002

[22] B. Wang, Y. Li, N. Wu and C. Lan. " $\mathrm{CO}_{2}$ bio-mitigation using microalgae". Applied Microbiology and Biotechnology. Vol. 79, pp. 707-718. May, 2008. ISSN: 01757598. URL: http://link.springer.com/ article/10. 1007/s00253-008-1518-y. DOI: 10.1007/ s00253-008-1518-y.10.1007/ s00253-008-1518-y. 